\title{
Classifying Pseudomonas aeruginosa Strains of Human Origin in MDR, XDR and PDR by Determining the Resistance to Antibiotics from Seven Groups
}

\author{
Oana - Alexandra CIOCAN (MOȚCO) ${ }^{1 *}$, Mihai CARP - CĂRARE ${ }^{1}$, Carmen - Valentina PANZARU ${ }^{2}$, \\ Elena PETRARU ${ }^{3}$ \\ ${ }^{1}$ Faculty of Veterinary Medicine, Iasi \\ ${ }^{2}$ Institute of Cardiovascular Diseases „Prof. Dr. George I.M. Georgescu”, Iasi \\ ${ }^{3}$ Emergency Hospital for Children „St. Mary”, Iasi \\ ${ }^{*}$ Corresponding author, e-mail: veterinarians_Phd@yahoo.com \\ Bulletin UASVM Veterinary Medicine 71(2) / 2014, \\ Print ISSN 1843-5270; Electronic ISSN 1843-5378 \\ DOI:10.15835/buasvmcn-vm: 10644
}

\begin{abstract} MDR, XDR and PDR strains were found in this study.

Keywords: antibiotics, Pseudomonas aeruginosa, $M D R, P D R, X D R$

\section{INTRODUCTION}

MDR has been defined as acquired nonsusceptibility to at least one agent in three or more antimicrobial categories, XDR has been defined as non-susceptibility to at least one agent in all but two or fewer antimicrobial categories (i.e. bacterial isolates remain susceptible to only one or two categories) and PDR has been defined as non-susceptibility to all agents in all antimicrobial categories. In order to ensure adequate application of these definitions, it is recommended that bacterial isolates are tested against all or nearly all of the antimicrobial agents within the antimicrobial categories and selective reporting and suppression of results should be avoided (Magiorakos et al., 2012).
\end{abstract}

Thirty-one strains of Pseudomonas aeruginosa of human origin from The Institute of Cardiovascular Disease "Prof. Dr. George I. M. Georgescu" Iasi were tested by disc diffusion method. According to the results, the strains were classified as MDR, XDR or PDR. The recent recommendations by Magiorakos et al., 2012 were used to define

The quality control of the study was carried out with Pseudomonas aeruginosa strain ATCC 27853.

A number of 26 of the 31 tested strains were found as MDR, 5 strains were included in the XDR group, no PDR

\section{MATERIALS AND METHODS}

Antibiotic susceptibility testing . Antibiotic sensitivity of strains included in the study was tested by disc diffusion method. Interpretation
Tab. 1. Antibiotics tested

\begin{tabular}{cc}
\hline Group of antibiotics & Antibiotic tested \\
\hline \multirow{2}{*}{ AMINOGLICOZIDE } & TOBRAMYCIN \\
\cline { 2 - 2 } & AMIKACIN \\
\cline { 2 - 2 } CARBAPENEME & GENTAMICIN \\
\cline { 2 - 2 } & IMIPENEM \\
\hline CEFALOSPORINE & MEROPENEM \\
\cline { 2 - 2 } & CEFTAZIDIME \\
\hline FLUOROQUINOLONES & CEFEPIME \\
\cline { 2 - 2 } & CIPROFLOXACIN \\
\hline PENICILINE+INHIBITORI & LEVOFLOXACIN \\
\hline DE $\beta$-LACTAMAZE & TAPERACILLIN- \\
\hline MONOBACTAME & AZTREONAM \\
\hline POLYMYXINE & COLISTIN \\
\hline
\end{tabular}


results we made based on the rules CLSI (Clinical and Laboratory Standard Institute). The seven groups of antibiotics that we used in this study were:

DST diffusion. Principle: the surface of Mueller-Hinton environment seeded with stem test cloth shall be submitted disks with different antibiotics; antibiotic diffuses circular medium at concentrations decreasing; bacterial growth is inhibited in the area where higher concentrations than MIC (Codiță et al., 2009).

Materials used: Strain tested in pure culture; Mueller-Hinton agar plates (Oxoid, UK); Discs impregnated with antibiotics (Oxoid, UK); Sterile saline; Sterile pads; 0.5 McFarland standard.

Working protocol: Mueller-Hinton medium I agar was prepared according to the manufacturer's instructions and poured into $25 \mathrm{ml}$ petri dishes with a diameter of $90 \mathrm{~mm}$, so that the thickness of the medium is $4 \mathrm{~mm}$. We prepared in $2 \mathrm{ml}$ of saline, a 0.5 McFarland turbidity of the bacterial suspension with 24 of the culture of the test strain ha, which corresponds to an inoculum of $1-2 \times 10^{8} \mathrm{CFU} / \mathrm{ml}$; suspension should be used within 15 minutes of mixing. Mueller-Hinton agar plates were seeded (pre-dried in the thermostat at 5 to 10 minutes) with a sterile sampling swab soaked in the bacterial suspension in three directions so as to spread out over the entire surface to obtain a confluent growth (Buiuc et al., 2010). The antibiotic discs were applied with automatically dispensaries Oxoid, UK. The plates were incubated at $37^{\circ}$, aerobically overnight (20$24 \mathrm{~h})$. The diameter of inhibition zones ( $\mathrm{mm}$ ) was measured around the discs and a category sensitivity (sensitive resistant, intermediate) were played according to CLSI standards. Antimicrobial agents and points of rupture zones of inhibition for Ps. aeruginosa (Performance Standards for Antimicrobial amended Susceptibility Testing; Twentieth Informational Supplement. CLSI document M 100 - S20, Wayne, PA, USA, 2014).

\section{RESULTS AND DISCUSSIONS}

- 31 the number of strains tested were 26 MDR (multidrug-resistant, the lack of sensitivity

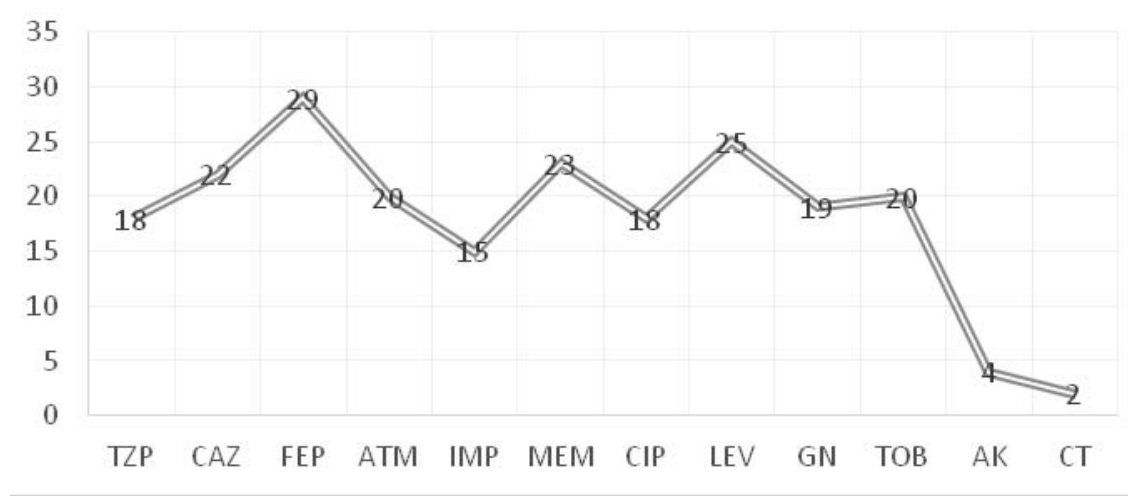

Fig. 1. Numerical strains with different resistance profiles

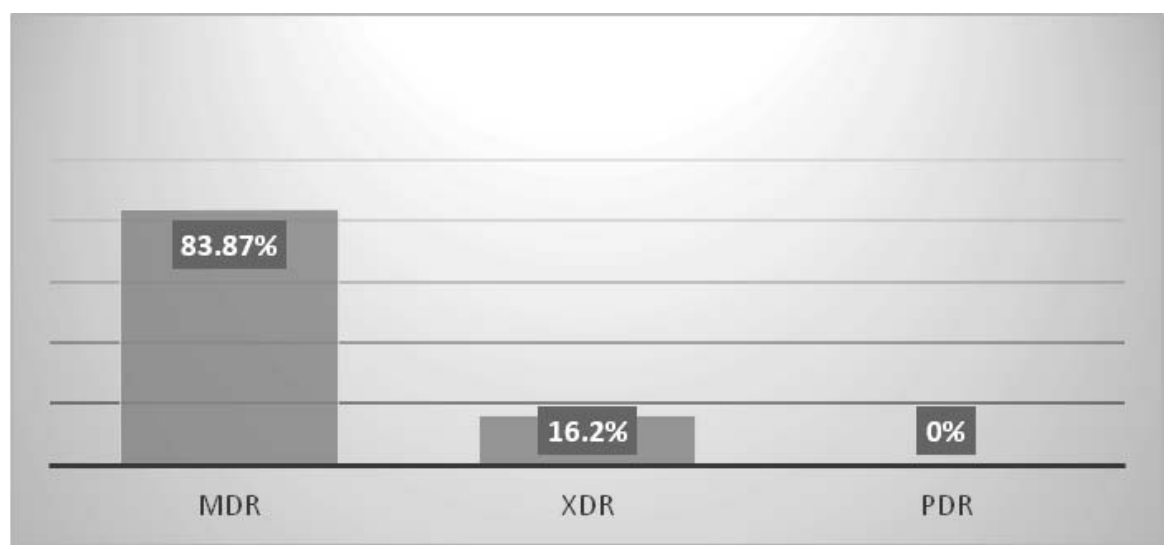

Fig. 2. Classification of strains of Pseudomonas aeruginosa in the MDR, XDR, PDR 
of at least one agent of 3 or more classes of antibiotics)

- 5 strains were included in the group XDR (extensively drug-resistant lack of sensitivity of at least one agent in all but one or two classes of antibiotics)

- in this study did not find any strain PDR (pandrugresistant, lack sensitivity all agents of all classes of antibiotics)

Bacterial isolates that are MDR will have many different resistance profiles because, by definitions, non-susceptibles results for even a single agent in only three agent antimicrobial categories defines an organism as MDR. (Aloush V. et al., 2006)

Panels of list of antimicrobial agents were developed for each organism group, as proposed harmonized templates that could be used by clinical, reference and public health microbiology laboratories that perform in vitro antimicrobial susceptibility testing, and wish to identify MDR, XDR and PDR. (Nihal Martis et al., 2014)

Pseudomonas aeruginosa MDR may result from the gradual acquisition of several mechanisms, as the horizontal transfer of genes, gene mutations and chromosomal (Magiorakos AP et al. 2012)

Antimicrobial resistance of the pathogens responsible for a majority of nosocomial infections continue to increase throughout the healthcare system. (Lautenbach E. et al., 2007)

\section{CONCLUSIONS}

1. This study did not reveal any strain pandrug resistant (PDR).
2. The highest sensitivity in this study is to Colistin.

3. DST diffusion is simple in execution, but complicated interpretation of results; interpretive reading and application of expert rules allow conversion of intermediate results recorded as sensitive or resistant, according to possible failures.

4. Lack of new antibiotics active against Pseudomonas aeruginosa, make the most important infection control measure against MDR strains of Pseudomonas aeruginosa.

\section{REFERENCES}

1. Aloush V, Navon-Venezia S, Seigman Igra Y, Cabili S, Carmeli Y (2006). Multidrug-resistant Pseudomonas aeruginosa: risk factors and clinical impact. Antimicrob Agents Chemother 50: 43-48.

2. Buiuc D, Negut M (2010). Treaty of Clinical Microbiology. Third edition, Medical Publishing House, Bucharest.

3. Codiță I, Buiuc D (2009). Determination of antibiotic: qualitative techniques. In Buiuc D Negut M (eds) Treaty of Clinical Microbiology. Third edition, Medical Publishing House, Bucharest.

4. Magiorakos AP, Srinivasan A, RB Carey et al. (2012). Multidrug-resistant, extensively drug-resistant and bacteria-resistant pandrug: an international expert Proposal for interim standard definitions for Acquired resistance. Clin Microbiol Infect 18: 268-281.

5. Martis N, Leroy S, Blanc V (2014). Colistin in multi drug resistant Pseudomonas aeruginosa blood - stream Infections. Journal of Infection xx, 1-12.

6. Lautenbach E, Polk RE. Resistant Gram-negative bacilli - a neglected healthcare crisis? Am J Health Syst Pharm 2007; 64 (December (23 Suppl 14)): S3-21. 